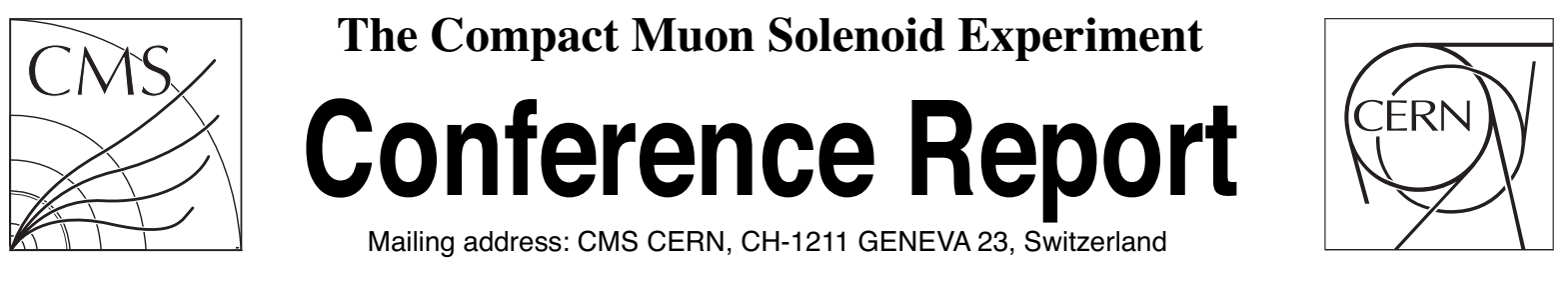

15 November 2011 (v2, 16 November 2011)

\title{
CMS Drift Tubes system during LHC 2010 operation
}

\author{
Cristina Fernandez Bedoya for the CMS Collaboration
}

\begin{abstract}
The DT (Drift Tube) chambers of the CMS (Compact Muon Solenoid) experiment are responsible for muon identification and precise momentum measurement. After several years of installation and commissioning, the DT system has finally been operated under proton-proton and heavy ions collisions in the LHC (Large Hadron Collider) at CERN during the 2010 campaign. Operation of the detector and its electronics during this period has been outstanding. More than $99 \%$ of the system has been fully operational and the downtime has been below $0.1 \%$ throughout the whole year. The operation of the trigger and data acquisition systems during this period has been remarkable. The main results summarizing the performance of the DT detector and its electronics with 2010 collision data are presented. Details about chamber and local trigger efficiency, calibration and synchronization are described, showing how well the challenging design goals have been met.
\end{abstract}




\title{
CMS Drift Tubes System during LHC 2010 Operation
}

\author{
Cristina Fernández Bedoya on behalf of the CMS collaboration
}

\begin{abstract}
The DT chambers of the CMS experiment are responsible for muon identification, precise momentum measurement and triggering. After several years of installation and commissioning, the DT system has finally been operated under proton-proton and heavy ions collisions in the LHC at CERN during the 2010 campaign. Operation of the detector and its electronics during this period has been outstanding. More than $99 \%$ of the system has been fully operational and the downtime has been below $0.1 \%$ throughout the whole year.

The operation of the trigger and data acquisition systems during this period has been remarkable. The main results summarizing the performance of the DT detector and its electronics with 2010 collision data are presented. Details about chamber and local trigger efficiency, calibration and synchronization are described, showing how well the challenging design goals have been met.
\end{abstract}

\section{INTRODUCTION}

CMS (Compact Muon Solenoid) [1] is a general purpose $\mathcal{C}_{\text {detector designed to run at the highest luminosity at the }}$ LHC collider. The central feature of the CMS apparatus is a superconducting solenoid of $6 \mathrm{~m}$ diameter that generates a magnetic field of up to 4 Tesla. Such a high field was chosen in order to allow the construction of a compact tracking system on its interior, and still performing good muon tracking on the exterior.

Muons are measured in CMS by means of three different technologies of gaseous detectors. In the barrel, where the magnitude of the residual magnetic field is of the order of 2 Tesla in the iron return yoke and the neutron background and muon rate are expected to be as low as a few $\mathrm{Hz} / \mathrm{cm}^{2}$, DTs (Drift Tubes) are used [2].

DT chambers are responsible for muon identification and precise momentum measurement over a wide range of energies. The DT system also provides a reliable and robust trigger system with precise bunch crossing assignment, complemented by a set of Resistive Plate Chambers (RPC) which provides redundancy in the trigger.

\section{The Drift Tube Detector}

The DT chambers are installed in the five wheels of the return yoke of the CMS magnet (named YB-2, YB-1, YB0, $\mathrm{YB}+1$ and $\mathrm{YB}+2$ ). Each wheel is divided in 12 sectors each covering $\sim 30^{\circ}$ around the interaction point and each sector is

Manuscript received November 15, 2011.

C. Fernández Bedoya is with CIEMAT, Avda. Complutense, 40, Edificio 22-P1-22, 28040 Madrid, Spain (telephone: +34-91-346-0859, e-mail: cristina.fernandez@ciemat.es). organized in four stations of DT chambers named MB1, MB2, MB3 and MB4 going from inside to outside, where MB stands for Muon Barrel. There are a total of 250 DT chambers in CMS. A schematic view of one CMS wheel is shown in Fig. 1.

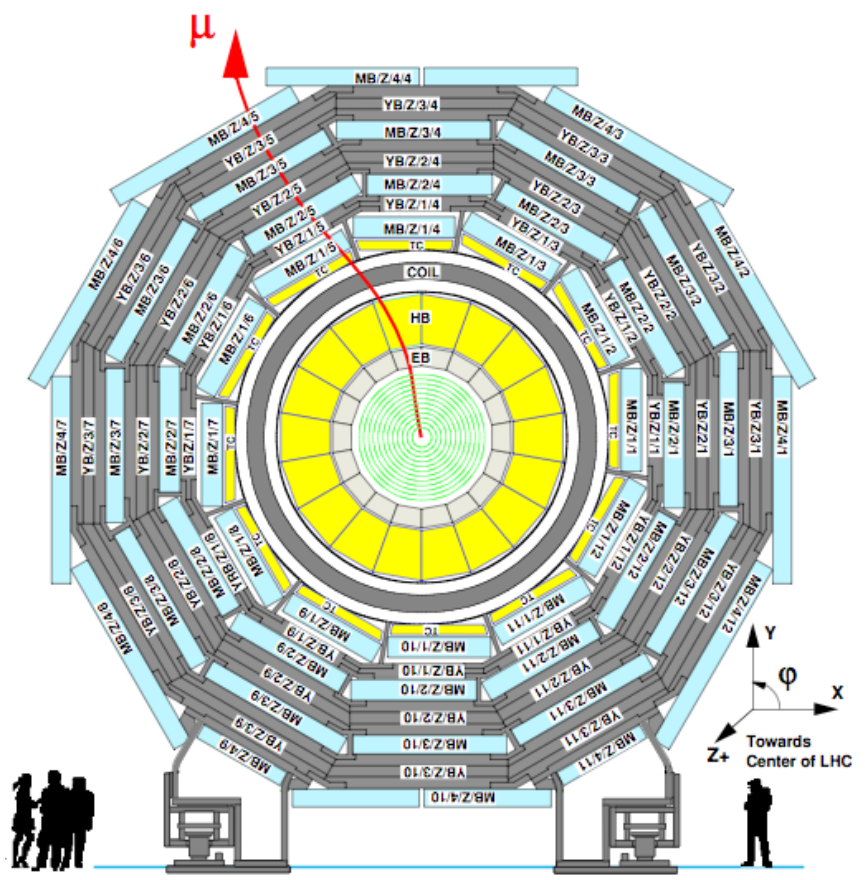

Fig. 1. Transverse view of a CMS Barrel Yoke wheel. The 12 sectors that subdivide each of the wheels are shown as well as the four concentric layers of DT chambers around the interaction point.

A DT chamber is made of three (or two in MB4) Superlayers (SL), each consisting of four layers of rectangular drift cells staggered by half a tube width. The wires in the two inner and outer SLs are parallel to the beam line and provide the track measurement in the magnetic bending plane $(\mathrm{r}, \Phi)$. In the central SL, the wires are orthogonal to the beam line and measure the position along the beam. This inner $\theta$ Superlayer is not present in the MB4 chambers, which therefore measure only the $\Phi$ coordinate.

The basic element of the DT chamber is the drift tube, which has cross section dimensions of $13 \mathrm{~mm} \times 42 \mathrm{~mm}$. The total number of sensitive cells is around 172,000. Any charged particle going through the cell volume will generate a signal (hit) in its anodic wire that will be amplified and discriminated by the front-end electronics for further time digitization. The position of the charged particle can be related to the time measurement since the drift velocity in the cell volume is 
approximately constant. The maximum drift time in each tube is around $400 \mathrm{~ns}$ and the spatial resolution that can be achieved at the cell level is in the order of $250 \mu \mathrm{m}$.

\section{A. DT Electronics}

One of the key features of the DT system is its robust and reliable trigger system, with precise bunch crossing assignment. (BX stands for bunch crossing, and refers to each 25 ns unit time in which collisions may occur in CMS.) This trigger system is based on the Mean-timer algorithm [3] and allows reconstructing tracks from the drift time measurements. For each BX the DT local trigger system provides up to two trigger segments per chamber in the $\Phi$ view, and one in the $\theta$ view. In the $\Phi$ view, each trigger segment is associated with the following quantities: the BX, at which the corresponding muon candidate was produced; the position and direction in the local coordinate system of the chamber; a quality word describing how many aligned DT hits were found; and a bit flagging the segment as a first or second candidate, ordered according to their assigned quality. One set of such quantities is called a "trigger primitive".

The trigger algorithm that provides the trigger primitives in the $\Phi$ view works in three logical steps that are sketched in Fig. 2. The Bunch and Track Identifiers (BTIs) search for track segment candidates made of three or four aligned DT hits within a SL in every BX. Each candidate is processed by the Track Correlators (TRACOs), which search for a proper angular matching between the candidates from the two SLs in each chamber. TRACO candidates are then sent to the Trigger Server, which performs a ghost-suppression mechanism and then selects the two trigger primitives with the highest quality.

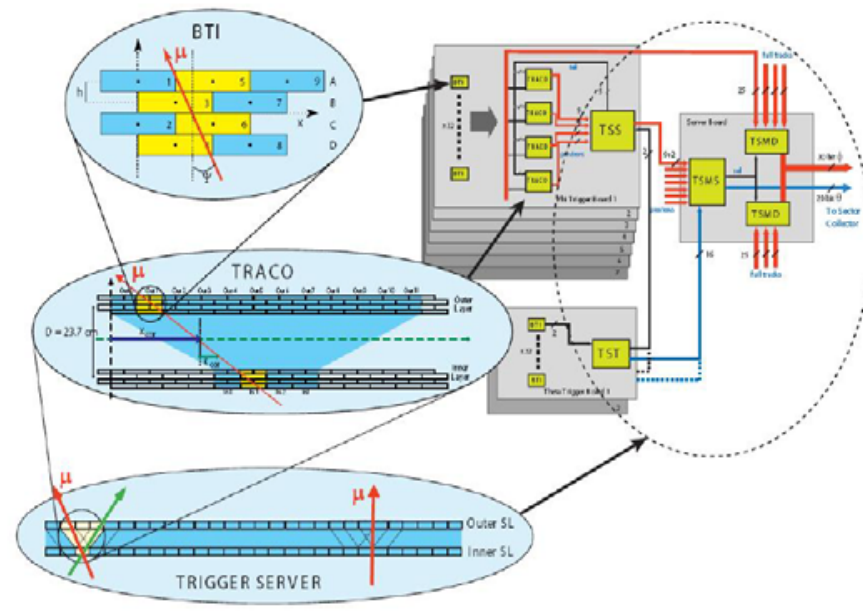

Fig. 2. The main components of the DT local trigger in the $\Phi$ view of a muon chamber. The BTIs detect hit alignments within each Superlayer, the TRACOs search for proper matching between SLs and the Trigger Server selects the best two candidates in the chamber and applies a ghost-suppression algorithm.

These two trigger primitives are sent to the Sector collector boards located in the CMS tower racks. They perform trigger synchronization and send the encoded information of position, transverse momentum and track quality through high-speed optical links to the DT Regional Trigger in the counting room. The Regional Trigger builds full muon tracks and forwards the best four muon candidates to the CMS Global Muon Trigger where information is combined with that of the other muon detectors [4].

\section{DT Muon Read-Out}

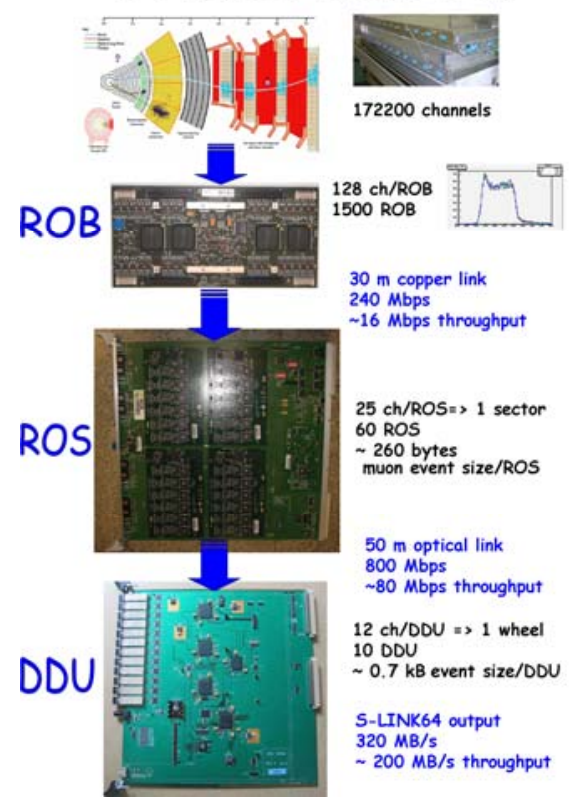

Fig. 3. Schematic view of the DT read-out chain with the ROB boards that perform the time digitalization and the ROS and DDU boards in charge of merging the information from the full detector. The DT read-out electronics applies various levels of data reduction and quality monitoring, building consistent event fragments synchronized with the events from the other CMS subdetectors.

In addition, the DT read-out electronics [5] is designed to perform time measurement of the chamber signals that will allow the reconstruction of charged particle tracks and precise momentum measurement. As can be seen in Fig. 3, there are several levels of data merging in order to achieve a read-out of the full detector at a Level-1 trigger rate of $100 \mathrm{kHz}$.

First elements are the ROBs (Read Out Boards), based on the ASIC HPTDC (High Performance Time to Digital Converter), that perform the time digitization of the hits and assign them to the Level 1 trigger. They transmit their data through a $\sim 30$ meter copper link to the 60 ROS (Read Out Server) boards located in the tower racks in the cavern. ROS boards are in charge of merging the information from one sector and perform several tasks of data reduction and data quality monitoring. Each sector event is retransmitted through an optical link to the DDU (Device Dependent Unit) boards located in the counting room. The DDU boards merge data from up to 12 ROS to build an event fragment and send it to the global CMS data acquisition system through an output at 320 MBps. 


\section{DT SYSTEM OPERATION AT LHC}

On March $30^{\text {th }} 2010$ LHC started proton-proton collisions at a center-of-mass energy of $7 \mathrm{TeV}$. Commissioning of the machine through all the year turned into a substantial increase of the maximum instantaneous luminosity, achieving up to $210^{32} \mathrm{~cm}^{-2} \mathrm{~s}^{-1}$ by the end of 2010. CMS operation has been remarkably smooth, with high data taking efficiencies that allowed to record $43.17 \mathrm{pb}^{-1}$ out of the $47.03 \mathrm{pb}^{-1}$ delivered by LHC. Operation of the DT detector during these more than 840 hours of operation in stable beams has been outstanding. The DT contribution to the total CMS downtime has been below $0.1 \%$ throughout the whole year. Major fraction of the DT downtime was due to the manual resynchronization command that allowed recovering blocked channels in the event of sporadic bursts of noise that fill up the read-out buffers or unlock the channels. Though the rate of these very noisy events is low, in the order of a few per week, an automatic resynchronization procedure was setup in August 2010 to speed up the recovery to few microseconds and since then, the downtime has been negligible.

On top of that, only one run (1 hour 30 minutes long) has been certified as bad during all the year due to an incident with a temperature sensor that triggered a false alarm and powered off one wheel during most of the run.

Out of the 172,000 channels of the DT detector, more than $99.5 \%$ have been operating properly through the entire data taking. Disconnected cells due to high voltage problems remain at the level of $0.1 \%$, and the loss in detector acceptance because of failures in the read-out and trigger electronics is below $0.4 \%$.

The DT Control and monitoring systems have also evolved as we have gained experience with operation under collisions. In particular we have tuned all the recovery mechanisms implementing several levels of alarms not only regarding safety aspects but also data quality problems.

In summary, stability and reliability of the DT detector has been very satisfactory and the failure rate of the electronics modules has been low and within our expectations. The main point of concern at present is due to the overheating of some low voltage connectors for which several actions are under study.

\section{DT DETECTOR PERFORMANCE}

\section{A. DT Trigger Performance}

The timing parameters of the LHC are such that proton bunches potentially collide inside CMS every $25 \mathrm{~ns}$ and the trigger system should identify the correct bunch crossing at which the corresponding signature (e.g. muon) was originated. Trigger synchronization [6] is of great importance to achieve this goal and it has to be controlled at three levels: intrachamber synchronization, chamber-to-chamber relative synchronization and subsystem-to-subsystem synchronization. The last two items depend on a coarse synchronization (in multiples of BXs) that was already achieved during cosmic rays data taking campaigns. However, a fine synchronization (with a precision of a few nanoseconds) with the LHC machine clock depends mainly on the muon time of flight from the interaction point to the chamber. Therefore, it was necessary to collect enough statistics of muons from protonproton collisions during the first months in order to perform this calibration. Up to four iterations have taken place based on the available statistics. Fig. 4 shows the resulting BX distribution of the local highest quality trigger primitives (i.e. built with at least 7 out of 8 layers in a chamber). Out-of-time primitives populate symmetrically the bins to the right and to the left of the correct BX, being the corresponding pre- and post-firing rates in the order of $2 \%$, in good agreement with the expectations [4].

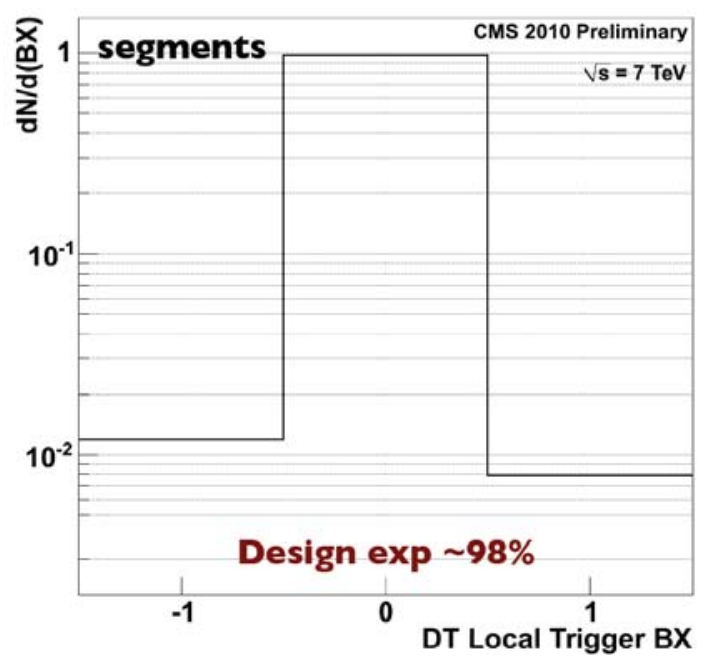

Fig. 4. Distribution showing the fraction of times in which the bunch crossing $(\mathrm{BX}=0)$ is correctly identified by the highest quality DT local trigger primitive. In this figure, data from all DT chambers are summed together.

The DT local trigger efficiency has also been studied. To perform this task, various requirements are set in samples of both minimum bias events and decays from $\mathrm{W}$ and $\mathrm{Z}$ bosons: selected events are required to be triggered by the RPC system without any requirement on the presence of a DT trigger; the presence of a track segment with at least 4 out of 8 hits and associated with the muon track is required in the same chamber; and finally, the selected segment has to be within the angular acceptance of the DT system. Results of the local trigger efficiency as a function of the transverse momentum, the $\Phi$ angle and the pseudorapidity are shown in Fig. 5, 6 and 7. (The pseudorapidity $\eta$ is defined as $-\ln [\tan (\theta / 2)]$, where $\theta$ is the polar angle of the particle with respect to the anticlockwise beam direction.)

The efficiencies shown take into consideration that the trigger primitive was found in the correct BX. As can be seen, the efficiencies are in the order of $95 \%$, which turns to be in very good agreement with the design expectations [4]. 


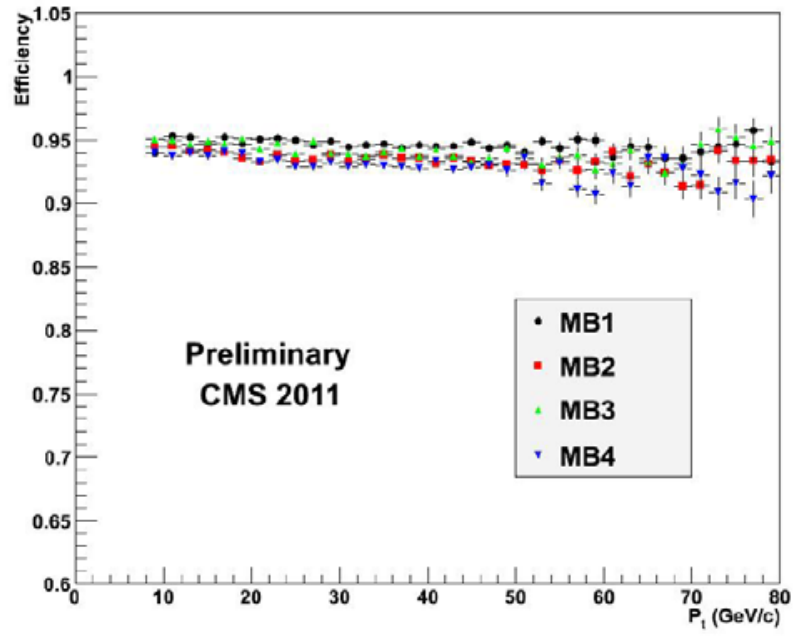

Fig. 5. DT local trigger efficiency as a function of the transverse momentum of the muon for the different types of chambers.

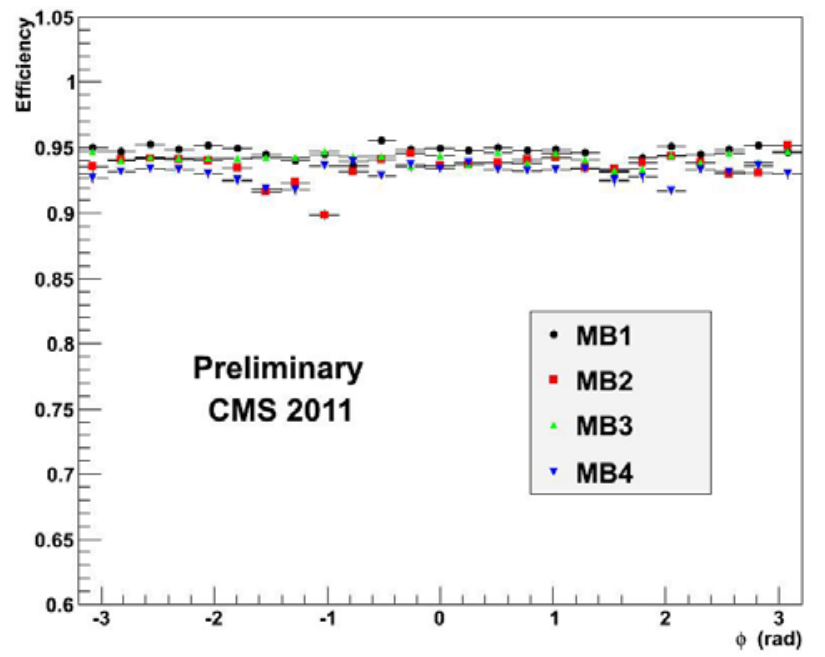

Fig. 6. DT local trigger efficiency as a function of the $\Phi$ angle of the muon for the different types of chambers. The drop in the MB2 for $\Phi \sim-1.2$ is due to a known problematic chamber.

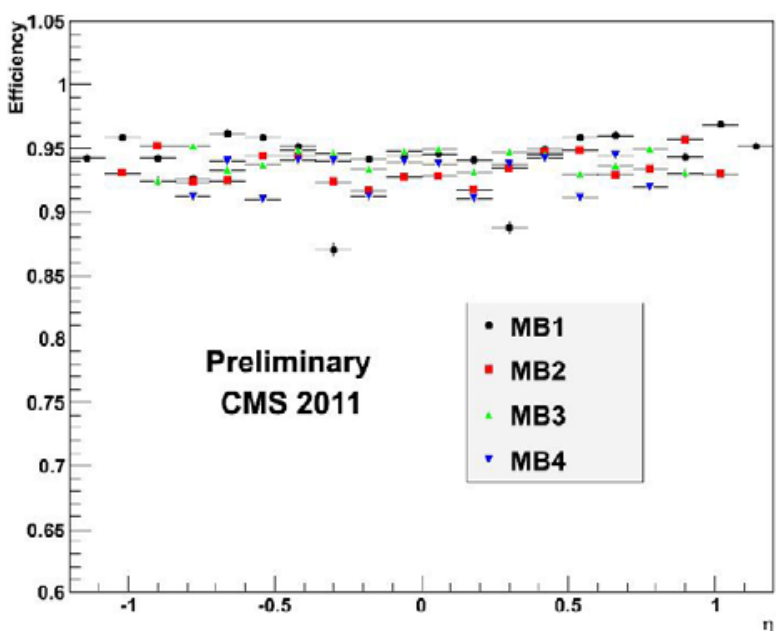

Fig. 7. DT local trigger efficiency as a function of the pseudorapidity of the muon for the different types of chambers.

\section{B. DT Resolution}

The main goal of the DT calibration is the determination of the relationship between the arrival time of the ionization signal and its spatial position. This includes the computation of the time pedestal to be subtracted to each cell drift time and the measurement of the drift velocity inside the chambers. This time pedestal takes into account contributions due to the inter-channel time difference due to the electronics inside the chamber, the Level 1 trigger latency, the time of flight of the muon and corrections due to the propagation time of the signal along the anode wire. Proper corrections have been performed to take into account the changes in the trigger timing configuration. The drift velocity obtained is around $55.5 \mu \mathrm{m} / \mathrm{ns}$ for all the $\mathrm{r}-\Phi$ Superlayers except for the MB1s of the external wheels where a lower value is observed because of the Lorentz angle induced by the stronger magnetic field.

The single hit position resolution achieved with the DT chambers depends strongly on the calibration parameters. Accordingly, the good values obtained reflect a good shape in the calibration of the detector.

The spatial hit resolution is determined from the distribution of hit residuals with respect to the reconstructed segments of the muon trajectory. The values achieved are shown in Fig. 8 and 9, and they are within the expectations. As can be seen in Fig. 8, the resolution of the outermost station (MB4) is slightly worst. This is due to the missing $\theta$ SL that allows in the other chambers obtaining the correction for the propagation along the wire. It is also to be noted the symmetry between the positive and the negative wheels values, and the fact that the resolution gets better in the external wheels due to the longer ionization patch inside the cell for muons with large $\theta$ angles. In the $\theta$ view, Fig. 9, it happens exactly the opposite way, since the effect corresponds to large impact angles in the cells.

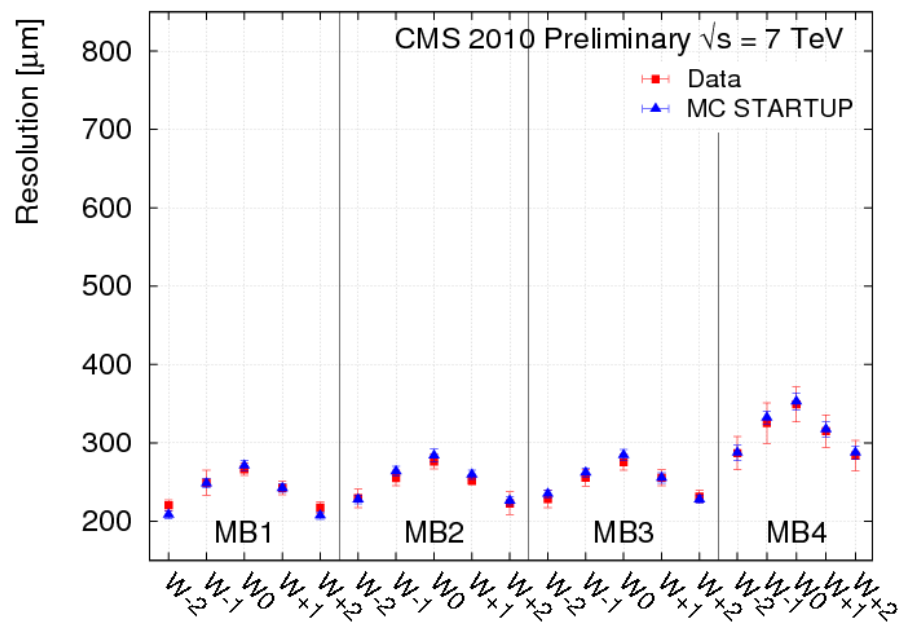

Fig. 8. Single-hit DT resolution for $\mathrm{r}-\Phi$ layers, averaged over all sectors in each barrel wheel and station. 


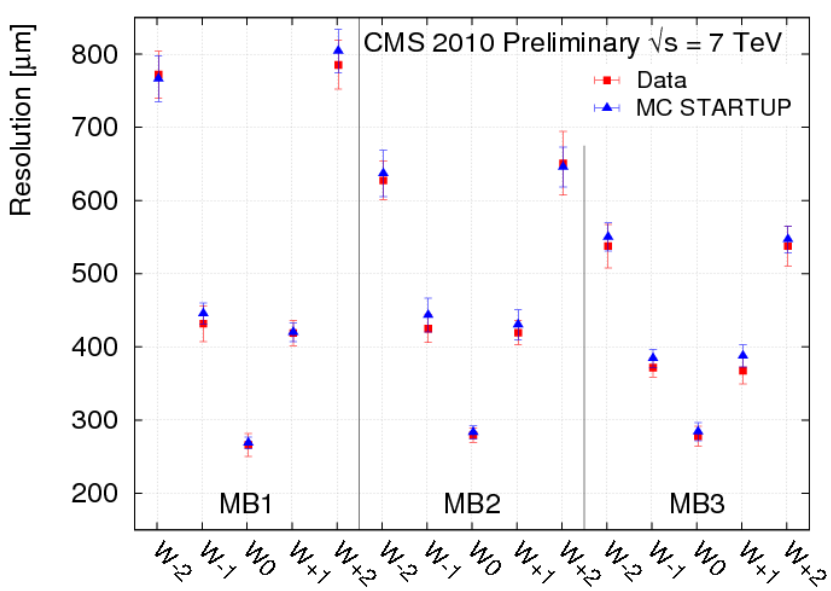

Fig. 9. Single-hit DT resolution for $\mathrm{r}-\theta$ layers, averaged over all sectors in each barrel wheel and station

In addition to the measurement of the track position and direction, DT provides a measurement of the arrival time of the track to the chamber. This calculation is performed by allowing a common displacement of all the hits to be a free parameter in the track segment fit. Assuming a constant drift velocity, the common displacement corresponds to a shift in time of the entire track. In Fig. 10 the distribution of the times for a high transverse momentum prompt muon sample can be seen.

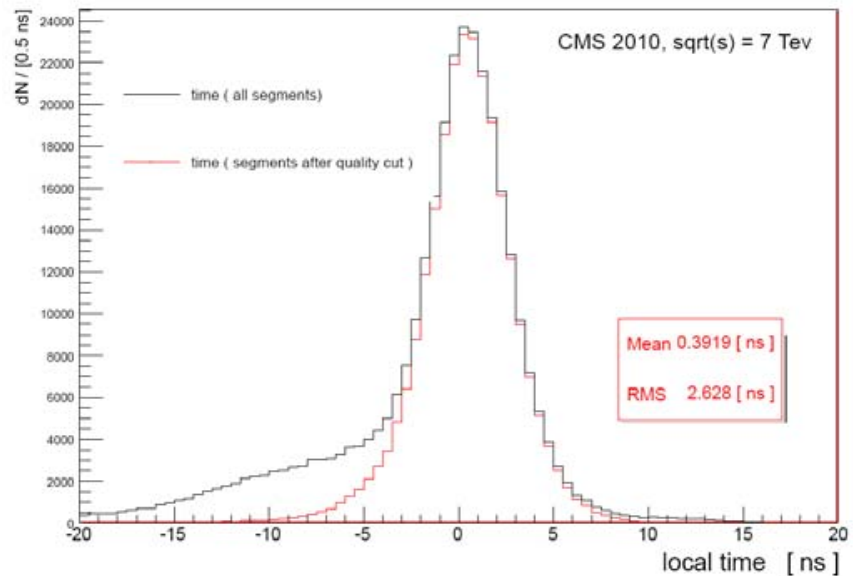

Fig. 10. Distribution of the local times as measured in the $r-\Phi$ projection of the DT chambers. The black distribution shows a clear tail due to the presence of delta-rays, while the red distribution shows the local time for segments that have passed a $\chi^{2}$ quality cut.

\section{DT Local Reconstruction Efficiency}

The local reconstruction efficiency, which refers to the track reconstruction within a chamber, has also been measured and compared to the Monte Carlo. For all the chambers, the values obtained are typically $90 \%$ or higher.

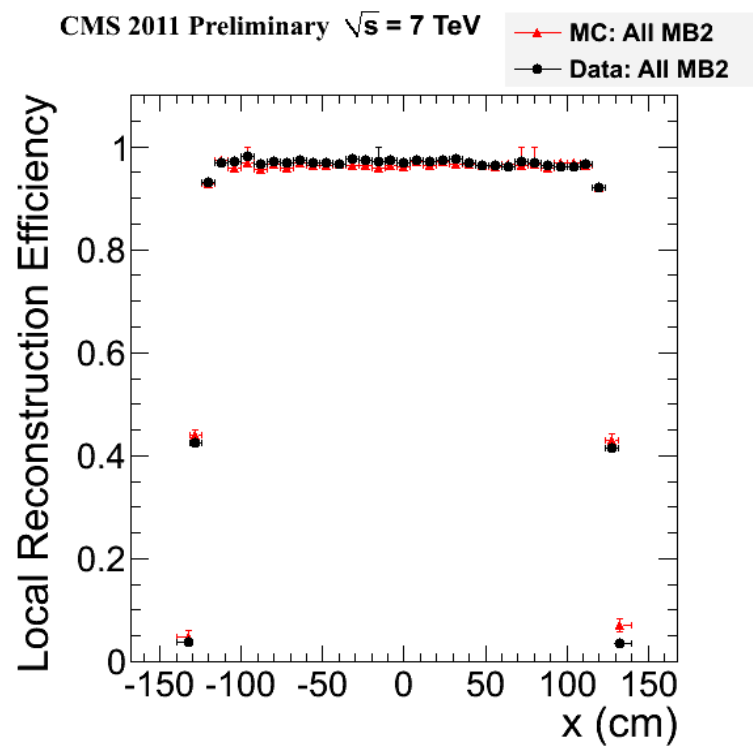

Fig. 11. Segment reconstruction efficiency in the MB2 DT chamber and comparison with simulated Monte Carlo data as a function of the local X coordinate in the chamber. Values have been integrated over all wheels and sectors.

The efficiency obtained versus the $\mathrm{x}$ coordinate transversal to the anodic wire inside the DT chamber is shown in Fig. 11. It can be seen how the observed efficiency matches very well the Monte Carlo expectations all the way to the edges of the chamber. Similar distributions have been obtained for all other barrel DT stations. Fig. 12 shows the segment efficiency as a function of $\eta$, where the lower efficiency in the separations of the central wheel can be observed.

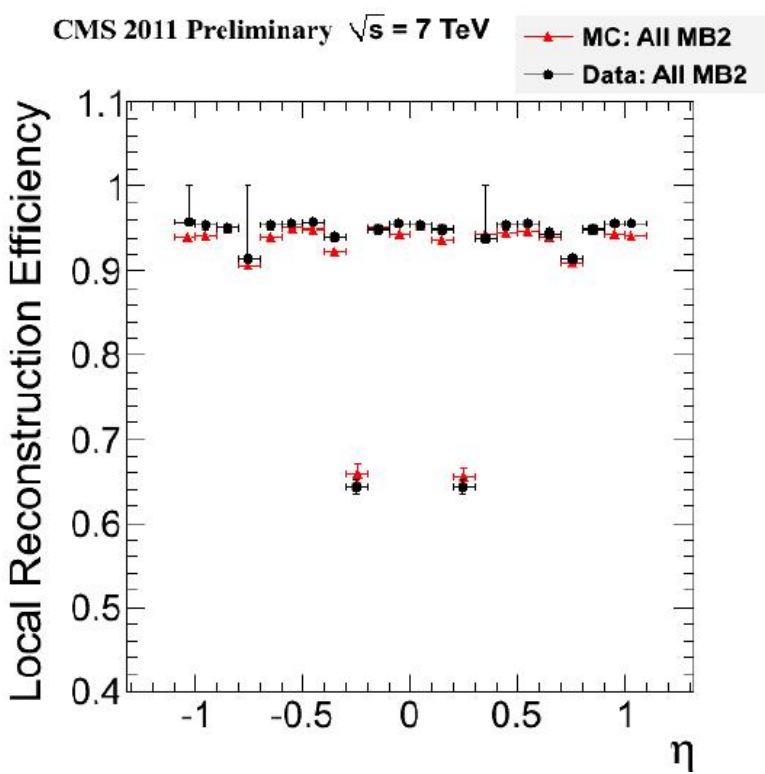

Fig. 12. Segment reconstruction efficiency in the MB2 DT chamber and comparison with simulated Monte Carlo data as a function of $\eta$. Values have been integrated over all wheels and sectors. No cut around the chamber edges has been applied in this plot. 


\section{DT Background}

Radiation background in the detector as a function of the luminosity has also been studied. Due to the low number $(\sim 20)$ of noisy cells $(>500 \mathrm{~Hz})$ in the DT chambers, and its stability versus time, the contribution of beam background can be disentangled easily from the intrinsic chamber noise. A non-track background sample by requiring only 1-2 hits within a Superlayer was studied (in comparison with the 3-4 hits obtained from a muon-like track). Since the DT system integrates over the full $1.25 \mu \mathrm{s}$ read-out window, with this method we have measured the rates of out-of-time backgrounds originated mainly from slow neutrons and punch-through activation as well as contamination from hits originated at other bunch crossings (pile up). The results show a clear linear trend as the luminosity increased, with values that seem to be in agreement with previous simulations [7].

The background rates for all the DT chambers are shown in Fig. 13, 14, 15 and 16. First thing one can infer from these plots is the effect of the shielding of the barrel wheel's steel, which reduces significantly the background in the MB2 chambers (Fig. 14) and even more in the MB3s (Fig. 15). Background in MB1 chambers appears to be mainly due to the leakage of particles from the hadron calorimeter and there is a strong dependence in $\eta$ as it is expected for background originating in collisions.

Instead, background in the MB4s is mainly due to the slow neutron gas that permeates the CMS cavern and that generates signals in the chamber at long times with respect to when the collision occurred. In Fig. 16 it can be seen that the background in MB4s is actually quite high, even higher than in the MB1 chambers. It can also be noted an important $\Phi$ asymmetry. This is due to the supports of the CMS wheels and the steel flooring of the CMS cavern.

In addition, the outer chambers of the YB-2 wheel observed approximately $20 \%$ higher background relative to the chambers located on the $\mathrm{YB}+2$ wheel. This effect positivenegative asymmetry seems to be due to the presence of the CASTOR detector on the minus side of the CMS.

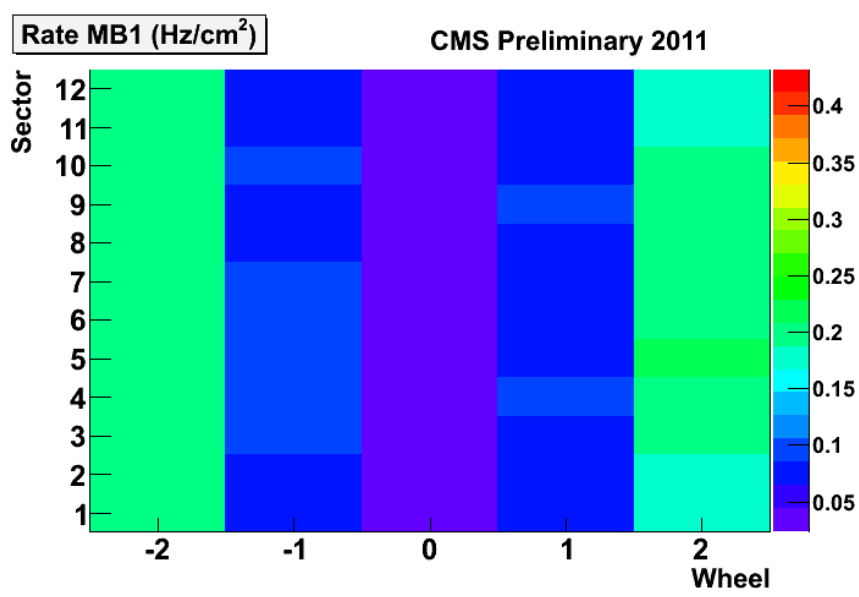

Fig. 13. Background rates of the MB1 DT chambers as a function of the wheel and of the sector the chamber it corresponds to. The measurement has been performed for an instantaneous luminosity of $10^{33} \mathrm{~cm}^{-2} \mathrm{~s}^{-1}$.

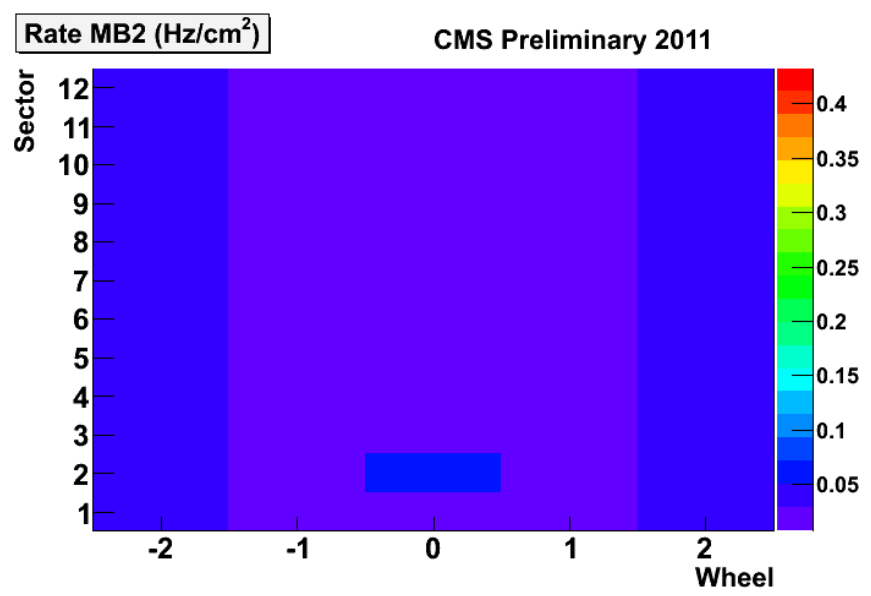

Fig. 14. Background rates of the MB2 DT chambers as a function of the wheel and of the sector the chamber it corresponds to. The measurement has been performed for an instantaneous luminosity of $10^{33} \mathrm{~cm}^{-2} \mathrm{~s}^{-1}$.

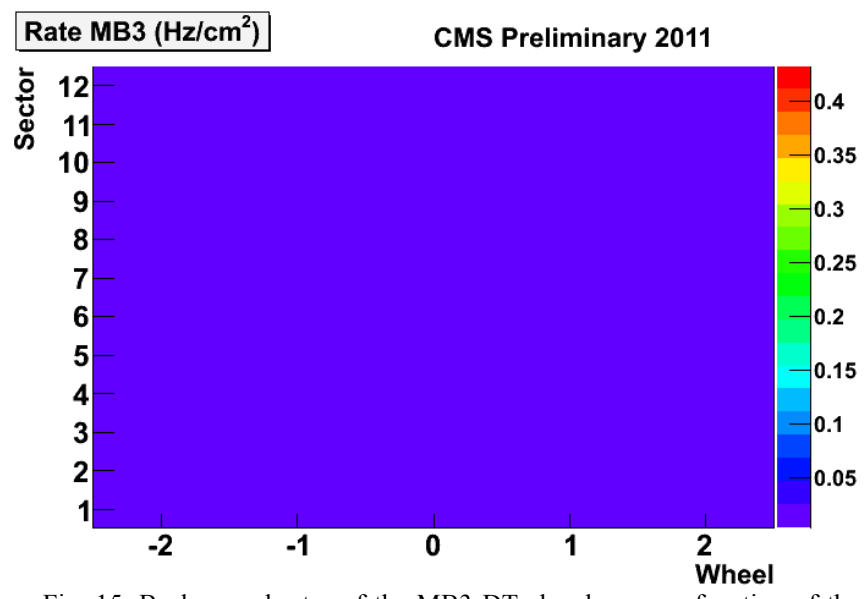

Fig. 15. Background rates of the MB3 DT chambers as a function of the wheel and of the sector the chamber it corresponds to. The measurement has been performed for an instantaneous luminosity of $10^{33} \mathrm{~cm}^{-2} \mathrm{~s}^{-1}$

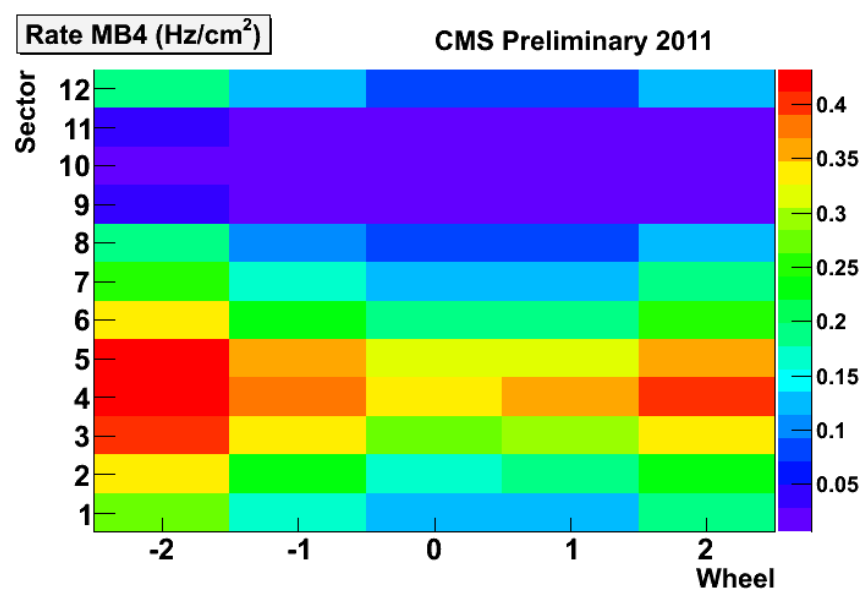

Fig. 16. Background rates of the MB4 DT chambers as a function of the wheel and of the sector the chamber it corresponds to. The measurement has been performed for an instantaneous luminosity of $10^{33} \mathrm{~cm}^{-2} \mathrm{~s}^{-1}$. 


\section{SUMMARY}

In summary, the performance of the DT system during LHC 2010 data taking has been outstanding, operating with very high efficiency and producing negligible downtime during CMS data taking. DT detector is an example of a very large and complex system that is working at present in a very robust and stable way through long periods of data taking.

The quality of the data acquired during LHC 2010 campaign is very good and has allowed studying the performance of the DT detector. Results presented here prove the high performance of the DT system not only as a triggering device but also for muon track reconstruction, meeting in both cases the design parameters. Measured background is also within expectations and does not represent nowadays a problem for operation and performance of the detector.

The data collected through this period have been very valuable for the physics analysis and represents an optimal starting point towards achieving the highest efficiency in reconstructing and identifying muons in LHC proton-proton collisions

\section{REFERENCES}

[1] CMS Collaboration, "The CMS experiment at the CERN LHC," JINST 3 S08004. 2008

[2] CMS Collaboration, “ The Muon Project Technical Design Report," CERN/LHCC 97-32 (1997).

[3] RD5 Collaboration, F. Gasparini et al. "Bunch crossing identification at LHC using a mean-timer technique." Nucl. Inst Methods Phys. Res. A, Vol. 336 (1993) 91.

[4] Trigger and Data Acquisition project, Volume I, "The Level-1 Trigger Technical Design Report," CERN/LHCC 2000-38, 2000.

[5] J.M. Cela, G. Dellacasa, C. Fernandez-Bedoya, J. Marin, V. Monaco, J.C. Oller, P. De Remigis, A. Staiano, C. Willmott, "CMS DT Chambers Read-Out Electronics," CMS CR 2008/018. CERN, 2008

[6] CMS collaboration, "Fine Synchronization of the CMS Muon DriftTube Local Trigger using Cosmic Rays," J. Instrum. 5 (2010) T03004.

[7] A. Benvenuti, V. Genchev. "Barrel Muon System Background Simulation of the CMS TDR Design," CMS NOTE 1998/052, CERN, 1998. 